\title{
Group Theoretic Technique for MHD Forced Convection Laminar Boundary Layer Flow of Nanofluid over a Moving Surface
}

\author{
Govind R. Rajput ${ }^{1 *}$, J.S.V.R. Krishnaprasad ${ }^{2}$ and M. G. Timol ${ }^{3}$ \\ ${ }^{* 1}$ SVKM's NMIMS, Mukesh Patel School of Technology Management and Engineering, Shirpur \\ Campus, Shirpur-425405, India \\ ${ }^{2}$ Department of Mathematics, M. J. College, Jalgaon-425001, India \\ ${ }^{3}$ Department of Mathematics, Veer Narmad South Gujarat University, \\ Magdulla Road, Surat - 395007, India \\ Email: g.rajput7@gmail.com
}

\begin{abstract}
In this paper steady two-dimensional forced convection laminar boundary layer viscous incompressible flow of nanofluid over a moving surface under the effect of magnetic field is studied. The similarity solution of the governing problem is obtained by using group theoretic method. The plate moves either in the same or the opposite direction to the free stream. The application of one-parameter transformation group that reduces the number of independent variables by one, is used to transform the model of governing partial differential equations into a system of ordinary differential equations with appropriate initial or boundary conditions.
\end{abstract}

Keywords: Nanofluid, MHD, Group theoretic technique, Natural convection.

\section{INTRODUCTION}

The forced convective heat transfer past a moving surface has applications in chemical processes, biochemical processes, solar receivers exposed to wind currents, cooling of reactors, heat exchangers, geothermal energy, oil extraction, oil exploration, plastic extrusion and continuous casting. Researchers are motivated towards its study because of the need to improve the efficiency of thermal conductivity and enhancing heat transfer which led to the utilization of nanoparticles in the fluid. The effect of temperature dependent viscosity on laminar mixed convective flow and heat transfer for a continuously moving vertical isothermal surface was studied by Ali [1], who obtained local similarity solutions. Klemp and Acrivos [2] investigated a method for integrating the boundary layer flow equations through a region of reverse flow past a parallel flat plate. Similar problems were studied by Ishak et al [3]. Magyari [4] studied the moving plate thermometer with uniform velocity for a series solution of the problem using the Merkin transformation method. The similarity solution for steady laminar boundary layer equations governing MHD flow near the forward stagnation point of two dimensional moving axisymmetric bodies was given by Hoernel [5].

Also it is very well known fact that the nanofluids can tremendously enhance the heat transfer characteristic of the base fluid. Therefore, nanofluids have great importance in the industry as coolants, lubricants and micro channel heat sinks. Choi [6] introduced the concept of the nanofluid. Choi et al. [7] proved that the addition of a very small amount that is one per-cent by volume of nanoparticles to pure fluids increases the thermal conductivity of the fluid by up to two. Bachok et al. [8] studied nanofluid forced convection boundary layer flow over a moving plate numerically and concluded that dual solutions exist when the plate and the free stream move in opposite directions. Hamad [9] presented an analytical solution for convection flow of a nanofluid over a stretching plate. Rajput et al. [10] derived similarity solution of MHD boundary layer flow of an electrically conducting, viscous incompressible nanofluid over a moving surface in the presence of a uniform magnetic field with thermal radiation. Khan and Pop [11] investigated the boundary layer flow and heat transfer of nanofluid over a stretching surface. AbdElGaied and Hamad [12] studied the problem of heat transfer in MHD forced convection flow of alumina-water nanofluid over moving permeable vertical flat plate with convective surface boundary condition. They found that alumina nanoparticles when suspended in a fluid are capable of increasing the heat transfer capability and the shear stress of the base fluid. In addition they found that the heat transfer rate increases with nanoparticle volume fraction. Hamad et al. [13] presented the effect of magnetic field on free convection flow of a nanofluid past a vertical semi-infinite flat plate and they showed that copper and mercury nanoparticles give better cooling performance compared with titanium oxide $\left(\mathrm{TiO}_{2}\right)$ and aluminum oxide $\left(\mathrm{Al}_{2} \mathrm{O}_{3}\right)$ particles, the advantage is not impressive enough. They also found that the thickness of thermal boundary layer increases with both the solid volume fraction of nanofluid and the magnetic parameter. Makinde and Aziz [14] studied nanofluid boundary layer 
flow over a stretching sheet with a convective boundary condition.

The aim of the the present study is to analyse, steady twodimensional laminar MHD forced convection flow of a nanofluid past a moving surface with convective surface boundary condition. The similarity solution is obtained by one parameter group of transformation.

\section{PROBLEM FORMULATION}

Consider two-dimensional forced convective flow of nanofluid over a moving flat plate. A rectangular coordinate frame is selected in which the $x$-axis is vertical and $y$-axis is horizontal. It is assumed that the plate moves with the constant velocity. The flow is takes place at $y \geq 0$ where $y$ is the coordinate measured normal to the moving surface. A uniform transverse magnetic field is applied at $y$-axis. Also assumed that, at the surface, temperature $\mathrm{T}$ and the nanoparticles fraction $\mathrm{C}$ take constant values $T_{w}$ and $C_{w}$ respectively whereas the values of $\mathrm{T}$ and $\mathrm{C}$ when $\mathrm{y}$ tends to infinity are denoted by $T_{\infty}$ and $C_{\infty}$ respectively.

The governing equations of the flow under consideration are given by,

$$
\frac{\partial \bar{u}}{\partial \bar{x}}+\frac{\partial \bar{v}}{\partial \bar{y}}=0
$$

$\bar{u} \frac{\partial \bar{u}}{\partial \bar{x}}+\bar{v} \frac{\partial \bar{u}}{\partial \bar{y}}=-\frac{1}{\rho_{n f}} \frac{\partial \bar{p}}{\partial \bar{x}}+\frac{\mu_{n f}}{\rho_{n f}} \frac{\partial^{2} \bar{u}}{\partial \bar{y}^{2}}-\sigma B^{2}(\bar{x}) \bar{u}$

$\bar{u} \frac{\partial T}{\partial \bar{x}}+\bar{v} \frac{\partial T}{\partial \bar{y}}=\alpha_{n f} \frac{\partial^{2} T}{\partial \bar{y}^{2}}$

$\bar{u} \frac{\partial C}{\partial \bar{x}}+\bar{v} \frac{\partial C}{\partial \bar{y}}=D_{m} \frac{\partial^{2} C}{\partial \bar{y}^{2}}$

Corresponding to the boundary conditions

$$
\begin{aligned}
& \bar{u}=\lambda \bar{u}_{w}(\bar{x}), \quad \bar{v}=0, \quad T=T_{w}, C=C_{w} \text { at } \bar{y}=0 \\
& \bar{u}=\bar{U}_{e}(\bar{x}), \quad T \rightarrow T_{\infty}, \quad C \rightarrow C_{\infty} \text { as } \bar{y} \rightarrow 0
\end{aligned}
$$

where $\bar{u}$ and $\bar{v}$ are the velocity components along $\bar{x}$ and $\bar{y}$ axes, $\bar{p}$ is the pressure, $\sigma$ is the electrical conductivity, $\mathrm{T}$ is the temperature of the nanofluid, $\bar{U}_{e}(\bar{x})$ is the potential velocity, $\bar{u}_{w}(\bar{x})$ is the velocity of the moving plate, $\rho_{n f}$ the density of base fluid, $\mu_{n f}$ is the nanofluid viscosity, $\alpha_{n f}$ is the nanofluid thermal diffusivity and $D_{m}$ is mass diffusivity and $\lambda$ is the constant moving wedge parameter with $\lambda>0$ corresponds to a moving wedge in the same direction and $\lambda<0$ corresponds to a moving wedge in the opposite directions to the free stream respectively, while $\lambda=0$ corresponds to a static wedge.

In stream function $\bar{u}=\bar{U}_{e}(\bar{x})$ so the equation (2) becomes

$$
\bar{U}_{e}(\bar{x}) \frac{d \bar{U}_{e}(\bar{x})}{d x}=-\frac{1}{\rho_{n f}} \frac{\partial \bar{p}}{\partial \bar{x}}-\frac{\sigma B^{2}(\bar{x}) \bar{U}_{e}(\bar{x})}{\rho_{n f}}
$$

Eliminating the pressure gradient term $\frac{\partial \bar{p}}{\partial \bar{x}}$ from equation (2) and (6), we get

$$
\begin{aligned}
\bar{u} \frac{\partial \bar{u}}{\partial \bar{x}}+ & \bar{v} \frac{\partial \bar{u}}{\partial \bar{y}}=\bar{U}_{e}(\bar{x}) \frac{d \bar{U}_{e}(\bar{x})}{d \bar{x}}+ \\
& \frac{1}{\rho_{n f}}\left[\mu_{n f} \frac{\partial^{2} \bar{u}}{\partial \bar{y}^{2}}-\sigma B^{2}(\bar{x})\left(\bar{u}-\bar{U}_{e}\right)\right]
\end{aligned}
$$

Introducing the following dimensionless boundary layer variables

$$
\begin{aligned}
& x=\frac{\bar{x}}{L}, y=\frac{\bar{y} \sqrt{\mathrm{Re}}}{L}, u=\frac{\bar{u}}{u_{\infty}}, v=\frac{\bar{v} \sqrt{\mathrm{Re}}}{u_{\infty}}, U_{e}=\frac{\bar{U}_{e}}{u_{\infty}}, \\
& \theta=\frac{T-T_{\infty}}{T_{w}-T_{\infty}}, \phi=\frac{C-C_{\infty}}{C_{w}-C_{\infty}}, S(x)=\frac{L}{u_{\infty}} \bar{S}(\bar{x})
\end{aligned}
$$

where $\mathrm{L}$ is the characteristic length, $u_{\infty}$ is the reference velocity, $\bar{S}(\bar{x})=\frac{\sigma B^{2}(\bar{x})}{\rho_{n f}}$ is the magnetic parameter, $\operatorname{Re}=\frac{u_{\infty} L}{v_{n f}}$ is the Reynolds number.

Introducing the stream function $\psi(x, y)$ defined by $u=\frac{\partial \psi}{\partial y}, v=-\frac{\partial \psi}{\partial x}$ so that it reduces the number of dependent variables as well as the number of equations. With this, equation (1) is satisfied automatically and equations (3), (4) and (7) become

$$
\begin{aligned}
\frac{\partial \psi}{\partial y} \frac{\partial^{2} \psi}{\partial y \partial x}-\frac{\partial \psi}{\partial x} \frac{\partial^{2} \psi}{\partial y^{2}}= & \frac{\partial^{3} \psi}{\partial y^{3}}+U_{e} \frac{d U_{e}}{d x} \\
& -S(x)\left(\frac{\partial \psi}{\partial y}-U_{e}\right)
\end{aligned}
$$

$\frac{\partial \psi}{\partial y} \frac{\partial \theta}{\partial x}-\frac{\partial \psi}{\partial x} \frac{\partial \theta}{\partial y}=\frac{1}{P_{r}} \frac{\partial^{2} \theta}{\partial y^{2}}$

$\frac{\partial \psi}{\partial y} \frac{\partial \phi}{\partial x}-\frac{\partial \psi}{\partial x} \frac{\partial \phi}{\partial y}=\frac{1}{S_{c}} \frac{\partial^{2} \phi}{\partial y^{2}}$

and boundary conditions are

$$
\begin{aligned}
& u=u_{w} \lambda, \quad v=0, \quad \theta=1, \quad \phi=1 \quad \text { at } y=0 \\
& u=u_{e}, \quad \theta=\phi=0 \quad \text { as } \quad y \rightarrow \infty
\end{aligned}
$$




\section{SOLUTION OF THE PROBLEM}

We use a method of solution which depends on the application of one parameter continuous deductive group of transformation to the system of partial differential equations (9)-(12). Under this class, we initially form the subgroup of transformations, after which the two independent variables will reduce by one variable and the system of non-linear partial differential equations (9)-(12) will transform into ordinary differential equations in terms of the similarity variable $\eta$.

\subsection{The group systematic formulation}

The procedure is initiated with group $G$, a class of one parameter ' $a$ ' of the form

$$
G:\left\{\begin{array}{l}
\bar{x}=h^{x}(a) x+k^{x}(a) \\
\bar{y}=h^{y}(a) y+k^{y}(a) \\
\bar{\psi}=h^{\psi}(a) \psi+k^{\psi}(a) \\
\bar{U}_{e}=h^{U_{e}}(a) U_{e}+k^{U_{e}}(a) \\
\bar{S}=h^{S}(a) S+k^{S}(a) \\
\bar{\theta}=h^{\theta}(a) \theta+k^{\theta}(a) \\
\bar{\phi}=h^{\phi}(a) \phi+k^{\phi}(a)
\end{array}\right.
$$

where $h^{R}$ and $K^{R}$ are real valued and at least differentiable in their real argument ' $a$ '

\subsection{Invariance analysis}

To transform the differential equation, transformations of the derivatives are obtained from $\mathrm{G}$ via chain-rule operations

$$
\begin{aligned}
& \frac{\partial \bar{p}}{\partial \bar{i}}=\left(\frac{h^{p}}{h^{i}} \frac{\partial p}{\partial i}\right) \\
& \frac{\partial^{2} \bar{p}}{\partial \bar{i}^{2}}=\left(\frac{h^{p}}{\left(h^{i}\right)^{2}} \frac{\partial^{2} p}{\partial i^{2}}\right) \quad i=x, y
\end{aligned}
$$

where, $\mathrm{p}$ stands for $\psi, U e, S, \theta, \phi$.

Now equation (9) is invariantly transformed for some function $H_{1}(a)$ whenever,

$$
\begin{aligned}
& {\left[\left(\frac{\partial \bar{\psi}}{\partial \bar{y}} \frac{\partial^{2} \bar{\psi}}{\partial \bar{y} \partial \bar{x}}-\frac{\partial \bar{\psi}}{\partial \bar{x}} \frac{\partial^{2} \bar{\psi}}{\partial \bar{y}^{2}}\right)-\bar{U}_{e} \frac{d \bar{U}_{e}}{d \bar{x}}+\frac{\partial^{3} \bar{\psi}}{\partial \bar{y}^{3}}+\bar{S}\left(\frac{\partial \bar{\psi}}{\partial \bar{y}}-\bar{U}_{e}\right)\right]=} \\
& H_{1}(a)\left[\frac{\partial \psi}{\partial y} \frac{\partial^{2} \psi}{\partial y \partial x}-\frac{\partial \psi}{\partial x} \frac{\partial^{2} \psi}{\partial y^{2}}-\frac{\partial^{3} \psi}{\partial y^{3}}-U_{e} \frac{d U_{e}}{d x}+S(x)\left(\frac{\partial \psi}{\partial y}-U_{e}\right)\right]
\end{aligned}
$$

Substitution from (13) and (14) into (15) yields,

$$
\begin{aligned}
& {\left[\begin{array}{l}
\frac{h^{\left(\psi^{2}\right)}}{h^{x} h^{y}}\left(\frac{\partial \psi}{\partial y} \frac{\partial^{2} \psi}{\partial y \partial x}-\frac{\partial \psi}{\partial x} \frac{\partial^{2} \psi}{\partial y^{2}}\right)-h \\
+\frac{h^{\psi}}{h^{\left(y^{3}\right)}} \frac{\partial^{3} \psi}{\partial y^{3}}+h^{S} \frac{h^{U}}{h^{x}} \frac{d U_{e}}{d x} \\
\left.h^{y} \frac{\partial \psi}{h^{y}}-h^{U} U_{e}\right)
\end{array}\right]+} \\
& R_{1}(a)=H_{1}(a)\left[\begin{array}{l}
\frac{\partial \psi}{\partial y} \frac{\partial^{2} \psi}{\partial y \partial x}-\frac{\partial \psi}{\partial x} \frac{\partial^{2} \psi}{\partial y^{2}}-\frac{\partial^{3} \psi}{\partial y^{3}} \\
-U_{e} \frac{d U_{e}}{d x}+S(x)\left(\frac{\partial \psi}{\partial y}-U_{e}\right)
\end{array}\right]
\end{aligned}
$$

where

$$
\begin{aligned}
& R_{1}(a)=k^{S}\left(\frac{h^{\psi}}{h^{y}} \frac{\partial \psi}{\partial \mathrm{y}}-h^{U} U_{e}-k\right. \\
& \left.U_{e}\right)-h^{S} S_{k} U_{e} \\
& { }_{-k} U_{e} \frac{h^{U_{e}}}{h^{x}} \frac{d U_{e}}{d x}
\end{aligned}
$$

Now equation (10) is invariantly transformed for some function $H_{2}(a)$ whenever,

$$
\begin{gathered}
{\left[\left(\frac{\partial \bar{\psi}}{\partial \bar{y}} \frac{\partial \bar{\theta}}{\partial \bar{x}}-\frac{\partial \bar{\psi}}{\partial \bar{x}} \frac{\partial \bar{\theta}}{\partial \bar{y}}\right)-\frac{1}{P_{r}} \frac{\partial^{2} \bar{\theta}}{\partial \bar{y}^{2}}\right]=} \\
H_{2}(a)\left[\frac{\partial \psi}{\partial y} \frac{\partial \theta}{\partial x}-\frac{\partial \psi}{\partial x} \frac{\partial \theta}{\partial y}-\frac{\partial^{2} \theta}{\partial \mathrm{y}^{2}}\right]
\end{gathered}
$$

Substitution from (13) and (14) into (18) yields,

$$
\begin{aligned}
& {\left[\frac{h^{\psi} h^{\theta}}{h^{x} h^{y}}\left(\frac{\partial \psi}{\partial y} \frac{\partial \theta}{\partial x}-\frac{\partial \psi}{\partial x} \frac{\partial \theta}{\partial y}\right)-\frac{1}{P_{r}} \frac{h^{\theta}}{h^{\left(y^{2}\right)}} \frac{\partial^{2} \theta}{\partial y^{2}}\right]=} \\
& H_{2}(a)\left[\frac{\partial \psi}{\partial y} \frac{\partial \theta}{\partial x}-\frac{\partial \psi}{\partial x} \frac{\partial \theta}{\partial y}-\frac{1}{P_{r}} \frac{\partial^{2} \theta}{\partial y^{2}}\right]
\end{aligned}
$$

Also equation (11) is invariantly transformed for some function $H_{3}(a)$ whenever,

$$
\begin{gathered}
{\left[\frac{\partial \bar{\psi}}{\partial \bar{y}} \frac{\partial \phi}{\partial \bar{x}}-\frac{\partial \psi}{\partial \bar{x}} \frac{\partial \phi}{\partial \bar{y}}-\frac{1}{S_{c}} \frac{\partial^{2} \phi}{\partial \bar{y}^{2}}\right]=} \\
H_{3}(a)\left[\frac{\partial \psi}{\partial y} \frac{\partial \phi}{\partial x}-\frac{\partial \psi}{\partial x} \frac{\partial \phi}{\partial y}-\frac{1}{S_{c}} \frac{\partial^{2} \phi}{\partial y^{2}}\right]
\end{gathered}
$$

Substitution from (13) and (14) into (19) yields,

$$
\begin{array}{r}
{\left[\frac{h^{\psi}}{h^{y}} \frac{h^{\phi}}{h^{x}}\left(\frac{\partial \psi}{\partial y} \frac{\partial \phi}{\partial x}-\frac{\partial \psi}{\partial x} \frac{\partial \phi}{\partial y}\right)-\frac{1}{S_{c}} \frac{h^{\phi}}{h^{\left(y^{2}\right)}} \frac{\partial^{2} \phi}{\partial y^{2}}\right]=} \\
H_{3}(a)\left[\frac{\partial \psi}{\partial y} \frac{\partial \phi}{\partial x}-\frac{\partial \psi}{\partial x} \frac{\partial \phi}{\partial y}-\frac{1}{S_{c}} \frac{\partial^{2} \phi}{\partial y^{2}}\right]
\end{array}
$$

The invariance of equations (16), (19) and (21) together with boundary conditions (12), implies that 
$\frac{h^{\left(\psi^{2}\right)}}{h^{x} h^{y}}=\frac{h^{\left(U_{e}^{2}\right)}}{h^{x}}=\frac{h^{\psi}}{h^{\left(y^{3}\right)}}=\frac{h^{S} h^{\psi}}{h^{y}}=h^{U_{e}} h^{S}$

$k^{S}=k^{U_{e}}=k^{y}=k^{\theta}=k^{\phi}=0$

These gives

$h^{x}=h^{\left(y^{3}\right)}, \quad h^{\psi}=h^{\left(y^{2}\right)}, \quad h^{U_{e}}=h^{y}, \quad h^{S}=\frac{1}{h^{\left(y^{2}\right)}}$

$h^{\theta}=h^{\phi}=1$

Finally we summarize in a subgroup $G$ of the form

$G:\left\{\begin{array}{l}\bar{x}=h^{\left(y^{3}\right)}(a) x+k^{x}(a) \\ \bar{y}=h^{y}(a) y \\ \bar{\psi}=h^{\left(y^{2}\right)}(a) \psi+k^{\psi}(a) \\ \bar{U}_{e}=h^{y}(a) U_{e} \\ \bar{S}=h^{\frac{1}{\left(y^{2}\right)}}(a) S \\ \bar{\theta}=\theta \\ \bar{\phi}=\phi\end{array}\right.$

This group transforms invariantly the differential equations (9) - (11) and the initial and boundary conditions (12).

\subsection{The complete set of invariants}

The complete set of absolute invariants are:

(i) The absolute invariants of the dependent variables $(x, y)$ are $\eta=\eta(x, y)$.

(ii)The absolute invariants of the dependent variables $\left(\psi, \mathrm{U}_{e}, S, \theta, \phi\right)$

$g_{j}\left(x, y: \psi, \mathrm{U}_{e}, S, \theta, \phi\right)=F_{j}(\eta(x, y)), j=1,2,3,4,5$.

Using the application of a basic theorem in group theory, (See Moran and Gaggioli [15]; Morgan [16]), i.e. A function $g\left(x, y: \psi, \mathrm{U}_{e}, S, \theta, \phi\right)$ is an absolute invariant of a one-parameter group if it satisfies the following first-order linear partial differential equation,

$\sum_{i=1}^{7}\left(\alpha_{i} Q_{i}+\beta_{i}\right) \frac{\partial g}{\partial Q_{i}}=0$

where

$Q_{i}=x, y, \psi, \mathrm{U}_{e}, S, \theta, \phi$

$\alpha_{i}=\frac{\partial h^{Q_{i}}}{\partial a}\left(a_{0}\right)$

$\beta_{i}=\frac{\partial k^{Q_{i}}}{\partial a}\left(a_{0}\right) \quad i=1,2,3,4,5,6,7$.

where $a_{0}$ denotes the value of parameter ' $a$ ' which yield the identity element of the group.

\subsection{Absolute invariants of the independent variables}

Since $k^{S}=k^{U_{e}}=k^{y}=k^{\theta}=k^{\phi}=0$ which gives $\beta_{2}=\beta_{4}=\beta_{5}=\beta_{6}=\beta_{7}$ and from equation (25) we get the following relations

$\alpha_{1}=3 \alpha_{2}=\frac{3}{2} \alpha_{3}=3 \alpha_{4}=-\frac{3}{2} \alpha_{5}, \quad \alpha_{6}=\alpha_{7}=0$

The absolute invariant $\eta(x, y)$ of the independent variables $(x, y)$ is determined using equation (24) if it will satisfy the first order linear partial differential equation

$\left(\alpha_{1} x+\beta_{1}\right) \frac{\partial \eta}{\partial x}+\alpha_{2} \frac{\partial \eta}{\partial y}=0$

Dividing $\alpha_{1}$ by we get,

$(x+\beta) \frac{\partial \eta}{\partial x}+\frac{y}{3} \frac{\partial \eta}{\partial y}=0, \quad$ where $\beta=\frac{\beta_{1}}{\alpha_{1}}$

Now, equation (27) can be written in the form

$\eta=y(x+\beta)^{\frac{-1}{3}}$

Further, for the absolute invariants of dependent variables owing the equation (24) are given by

$g_{1}(x, y, \psi)=\frac{\psi+\beta^{\prime}}{(x+\beta)^{2 / 3}}=f(\eta)$

$g_{2}\left(x, y, \mathrm{U}_{e}\right)=\frac{\mathrm{U}_{e}}{(x+\beta)^{1 / 3}}=\sigma_{2}(\eta)$

$g_{3}(x, y, \mathrm{~S})=\frac{S}{(x+\beta)^{-2 / 3}}=\sigma_{3}(\eta)$

$g_{4}(x, y, \theta)=\theta(\eta)$

$g_{5}(x, y, \phi)=\phi(\eta)$

Since $U_{e}(x)$ and $S(x)$ are functions $x$ of only and $\eta$ depends on $x$ and $y$ then $\Gamma_{2}(\eta)$ and $\bar{F}_{3}(\eta)$ must be constants, say $\mathrm{c}$ and $\mathrm{M}$.

Thus, finally we get the complete set of absolute invariants for the group $G$ which transforms the partial differential equations (9)-(11) into ordinary differential equation with boundary conditions (12) as

$\psi+\beta^{\prime}=f(\eta)(x+\beta)^{2 / 3}$
$\mathrm{U}_{e}=c(x+\beta)^{1 / 3}$
$S(x)=M(x+\beta)^{-2 / 3}$
$\theta=\theta$
$\phi=\phi(\eta)$

where $\eta(x, y)=y(x+\beta)^{-1 / 3}$ 


\subsection{Reduction to an ordinary differential equation}

Use above transformations (30) into equations (9)-(11), yields to nonlinear ordinary differential equations

$$
\begin{aligned}
& 3 f^{\prime \prime \prime}-f^{\prime 2}-M\left(f^{\prime}-c\right)+c^{2}=0 \\
& \frac{1}{P_{r}} \theta^{\prime \prime}+2 f \theta^{\prime}=0 \\
& \frac{1}{S_{c}} \phi^{\prime \prime}+\frac{2}{3} f \phi^{\prime}=0
\end{aligned}
$$

Now, to transform the boundary conditions into constant form, the free stream velocity must be of the form $u_{w}=(x+\beta)^{\frac{1}{3}}$. Then the transformed boundary conditions are

$$
\begin{aligned}
& f^{\prime}=\lambda, \quad f=0, \quad \theta=1, \quad \phi=1 \quad \text { at } \eta=0 \\
& f^{\prime}=c, \quad \theta=\phi=0 \quad \text { as } \eta \rightarrow \infty
\end{aligned}
$$

Here prime denotes the differentiation with respect to $\eta$. The parameters $P_{r}$ and $S_{c}$ are defined as:

$$
P_{r}=\frac{v}{\alpha_{n f}} \text { and } S_{c}=\frac{v}{D_{m}}
$$

It is very interesting to note the following deductions:

Taking the magnetic parameter $M=0$ and the constant $c=1$ in the differential equation (31)-(34) and by omitting equation (19), setting $S=0, m=\frac{1}{3}, N t=0$ in equations (16)(20) will reduce to that of obtained by [17].

Taking the magnetic parameter $M=0, \lambda=1$ and the constant $c=0$ in the differential equation (31)-(34) and setting $\varepsilon=0, b=1, m=0$ and $f w=0$ (the plate is impermeable) in equations (25)-(27) will reduce to that of obtained by [18].

\section{CONCLUSION}

Similarity solution of MHD forced convection laminar boundary layer flow of a nanofluid over a moving surface is studied by using one parameter group of transformation. The application of one parameter transformation group reduces the number of independent variables, by one, and consequently, the system of governing partial differential equations with boundary and initial conditions transforms into a system of ordinary differential equations with appropriate boundary and initial conditions.

\section{ACKNOWLEDGMENT}

Author's thanks to referee for constructive comments and suggestions.

\section{REFERENCES}

[1] Ali M. E., "The effect of variable viscosity on mixed convection heat transfer along a vertical moving surface," International Journal of Thermal Science, vol. 45, pp. 60-69, 2006. DOI: 10.1016/j.ijthermalsci.2005.04.006.

[2] Klemp, J. B. and Acrivos A., "A method for integrating the boundary-layer equations through a region of reverse flow," Journal of Fluid Mechanics, vol. 53, pp. 177-191, 1972. DOI: 10.1017/S0022112072000096.

[3] Ishak A., Nazar R., and I. Pop, "The effects of transpiration on the flow and heattransfer over a moving permeable surface in a parallel stream," Chem Eng J, vol. 148, pp. 63-67, 2009. DOI: 10.1016/j.cej.2008.07.040.

[4] Magyari E., "The moving plate thermometer," International Journal of Thermal Science, vol. 47, pp. 1436-1441, 2008. DOI: $\underline{\text { 10.1016/ }}$ j.ijthermalsci.2007.12.10.

[5] J. D. Hoernel, "On the similarity solutions for a steady MHD equation," Communications in Nonlinear Science and Numerical Simulation, vol. 13, no. 7, pp. 1353-1360, 2008. DOI: 10. 1016/j.cnsns.2006.10.010.

[6] Choi, S.U.S., "Enhancing thermal conductivity of fluids with nanoparticles" in D.A. Siginer, H.P. Wang (Eds.), Developments and Applications of NonNewtonian Flows, FED-V.231/MD-V.66, pp. 99-105, ASME, New York, NY, USA, 1995

Choi, S.U.S., Z. G. Zhang, W. Yu, F. E. Lockwood, and E. A. Grulke, "Anomalously thermal conductivity enhancement in nanotube suspension," Applied Physics Letters, vol. 79, pp. 2252-2254, 2001. DOI: 10.1063/1.1408272.

[8] Bachok. N., Ishak A., and I. Pop, "Boundary-layer flow of nanofluids over a moving surface in a flowing fluid," International Journal of Thermal Sciences, vol. 49, no. 9, pp. 1663-1668, 2010. DOI: 10.1016/j.ijthermalsci.2010.01.026.

[9] Hamad, M.A.A., "Analytical solution of natural convection flow of a nanofluid over a linearly stretching sheet in the presence of magnetic field," International Communications in Heat and Mass Transfer, vol. 38, no. 4, pp. 487-492, 2011. DOI: 10.1016/j.icheatmasstransfer.2010.12.042.

[10] Rajput G. R., Krishnaprasad, J.S.V.R. and M. G. Timol, "Application of scaling group transformation forMHD boundary layer flow and heat transfer of nanofluids over moving surface subject to suction/injection in the presence of thermal radiation with chemical reaction," Int. J Adv. Appl. Math. and Mech, vol. 3, no. 1, pp. 139-144, 2015.

[11] W. A. Khan and I. Pop, "Boundary-layer flow of a nanofluid past a stretching sheet," Int. J. Heat Mass Transfer, vol. 53, pp. 2477-2483, 2010. DOI: 10.1016/j.ijheatmasstransfer.2010.01.032.

[12] S. M. AbdEl-Gaied and Hamad, M.A.A, "MHD forced convection laminar boundary layer flow of aluminawater nanofluid over a moving permeable flat plate with convective surface boundary condition," Journal of Applied Mathematics, vol. 2013, 2013. DOI: $10.1155 / 2013 / 403210$.

[13] Hamad, M.A.A., I. Pop, A. I. Md Ismail, "Magnetic field effects on free convection flow of a nanofluid 
past a vertical semi-infinite flat plate," Nonlinear Analysis: Real World Applications, vol. 12, no. 3, pp. 1338-1346, 2011 .

DOI: 10.1016/j.nonrwa.2010.09.014.

[14] Makinde, O. D and Aziz A, "Boundary layer flow of a nanofluid past a stretching sheet with a convective boundary condition," International Journal of Thermal Sciences, vol. 50, no. 7, pp. 1326-1332, 2011. DOI: 10.1016/j.ijthermalsci.2011.02.019.

[15] Moran, M. J. and Gaggioli R. A, "Reduction of the number of variables in system of partial differential equations with auxiliary conditions" SIAM J Appl. Math, no. 16, pp. 202-215, 1968. DOI: $10.1137 / 0116018$.

[16] Morgan A.J.A., "The reduction by one of the number of independent variables in some systems of nonlinear partial differential equations," Quarterly Journal of Mathematics, Oxford 3, pp. 250-259, 1952.

[17] Zaimi Khairy, Anuar Ishak, and Ioan Pop. "Stagnation-point flow toward a stretching/shrinking sheet in a nanofluid containing both nanoparticles and gyrotactic microorganisms," Journal of Heat Transfer vol. 136. no. 4, pp. 041705, 2014. DOI: $10.1115 / 1.4026011$.

[18] Jalil Mudassar and Saleem Asghar, "Flow and heat transfer of powell-eyring fluid over a stretching surface: A lie group analysis." Journal of Fluids Engineering, vol. 135, no. 12 pp. 121201, 2013. DOI: $\underline{10.1115 / 1.4025097 .}$

\section{NOMENCLATURE}

B variable magnetic field

M dimensionless magnetic number

$\mathrm{T} \quad$ temperature of the fluid

$\mathrm{u} \quad$ velocity component along $\mathrm{x}$-axis

$v \quad$ velocity component along y-axis

$T_{\infty} \quad$ temperature of the fluid in the free stream

$T_{w}$
$\mathrm{C}$

$C_{w}$

$C_{\infty}$

c

$\operatorname{Pr}$

Re

$\mathrm{Dm}$

$\bar{u}_{w}(\bar{x})$

$\mathrm{p}$

\section{Greek symbols}

$\eta$

$\sigma$

$\theta$

$\phi$

$\mu_{n f}$

$\rho_{n f}$

$\alpha_{n f}$

$\lambda$

$\psi$

Subscripts

$\infty$

W

Superscripts nanoparticle volume fraction nanoparticle volume fraction at the surface

nanoparticle volume fraction in the free stream

constant

Prandtl number

Reynolds number

mass diffusivity

velocity of moving plate

pressure

dimensionless similarity variable

electrical conductivity

dimensionless temperature

dimensionless nanoparticle volume

fraction

nanofluid viscosity

density of the base fluid

nanofluid thermal conductivity constant moving wedge parameter stream function

condition at free steam

condition at the surface

differentiantion with respect to $\eta$ 